\title{
Relación entre actividad física, composición corporal e imagen corporal en estudiantes universitarios
}

\section{Relationship between physical activity, body composition and body image in university students}

\author{
Joana Cristina Serpa, Estefanía Castillo, Ana Paula Gama y Francisco Javier Giménez*
}

Universidad de Huelva (España)

\begin{abstract}
Resumen: Este trabajo tiene como objetivo principal analizar la relación entre la composición corporal, la imagen corporal y el nivel de actividad física de estudiantes universitarios. La descripción general de la muestra se realizó a través de las variables dependientes (sexo y edad), y por la identificación de diferentes parámetros de composición corporal (peso y altura). El análisis de la imagen corporal se desarrolla a través de sus dos vertientes, una perceptiva (Figure Rating Scale - Stunkard, Sorenson y Schlusinger, 1983), y otra subjetiva (Body Shape Questionnare - Cooper, Taylor, Cooper, y Fairburn, 1987). El nivel de actividad física se analizó a través del International Physical Activity Questionnaire (Craig et al., 2003). La muestra ( $\mathrm{n}=$ 400) está constituida por estudiantes universitarios, de los cuales, el 49,5\% son mujeres, y el 50,5\% son hombres, con una media de edad de 30,64 $( \pm 8,85$ ańos). Entre los resultados se puede destacar que la mayoría de los estudiantes tienen un nivel bajo de actividad física, y los sujetos que están más insatisfechos y más preocupados con su imagen corporal son los que se identifican con las siluetas más grandes.
\end{abstract}

Palabras clave: alumnado universitario, composición corporal, imagen corporal, actividad física.
Abstract: This work aims to analyze the relationship among body composition, body image and physical activity level in university students. The general description of the sample was made through the dependent variables (sex and age), and the identification of different body composition parameters (weight and height). The analysis of the body image was developed through its two components: perceptive (Figure Rating ScaleStunkard, Sorenson y Schlusinger, 1983), and a subjective one (Body Shape Questionnare-Cooper, Taylor, Cooper, y Fairburn, 1987). The PA level was analyzed through the International Physical Activity Questionnaire (Craig et al., 2003). The sample $(\mathrm{n}=400)$ was composed by university students, ( $49,5 \%$ women and $50,5 \%$ men) with a mean age of 30,64 ( \pm 8.85 years). Results highlight that most students have a low level of PA, and there is an inverse relationship between body composition parameters and body image, that is, subjects who identify themselves with the larger silhouettes are those who are most dissatisfied and more concerned with their body image. Keywords: university students, body composition, body image, physical activity.

\section{Introducción}

La imagen corporal se refiere a la representación mental del tamańo, figura y la forma de nuestro cuerpo, o sea, como nos vemos y como pensamos que los otros nos ven (Salazar, 2008). Además de la percepción, la imagen corporal implica igualmente la forma en la que sentimos el cuerpo y como actuamos sobre él. Así, se pueden estudiar dos aspectos de la imagen corporal: el perceptivo, referido a la percepción del tamaño corporal; y el subjetivo, que incluye las actitudes acerca del tamaño, peso, partes corporales y la apariencia física general (Cash y Pruzinsky, 1990). En la actualidad, el modelo estético corporal femenino es delgado, incluso excesivamente delgado, y enfatizando la apariencia sensual. Mientras que el ideal de belleza en los hombres es un patrón cultural musculado, con elevado grado de hipertrofia y niveles muy bajos de grasa corporal (Labre, 2002; Murnen, 2011).

En diferentes estudios se ha analizado la relación entre actividad física y parámetros de composición corporal (López Sánchez, López Sánchez, y Díaz Suárez, 2015; López Sánchez,

Dirección para correspondencia [Correspodence address]: Francisco Javier Giménez Fuentes-Guerra. E-mail: jfuentes@dempc.uhu.es
Borrego Balsalobre, y Díaz Suárez, 2013; López Sánchez, Nicolás López, y Díaz Suárez, 2016; López, Díaz, Radzimiński, \& Jastrzębski, 2017). Otros trabajos demuestran la relación positiva entre ejercicio físico e imagen corporal (Womack, 2010; Borrego, López, y Díaz, 2012; Borrego, López y Díaz, 2014; López, López y Díaz, 2015), contribuyendo la actividad física a tener evaluaciones más precisas sobre el propio cuerpo (Marques, 2003; Oliveira, 2009), y además un incremento de la satisfacción con la imagen corporal (Burgess, Grogan, y Burwitz, 2006, Grogan et al., 2014). También se ha relacionado la práctica de actividad física y la mejora de la satisfacción con la imagen corporal con la disminución de ansiedad física y social (Krane et al., 2001). Otros autores (Hart, 2000; Hausenblas y Fallon, 2006; Teixeira et al., 2006) indican que el ejercicio proporciona mejoras en el bienestar psicológico, autoestima y en la imagen corporal, siendo muchas veces ese el motivo principal para el inicio de la práctica deportiva. La actividad física parece ser una de las variables de mayor importancia en el desarrollo equilibrado de la persona (Castro et al., 2015; Chacón et al., 2016; Espejo et al., 2017). Se presenta como la vía obligatoria a seguir para la obtención 
de mayores niveles de satisfacción con la imagen corporal y la autoestima global, así como mayor responsabilidad por el propio cuerpo.

Russo (2005) estudia, en población universitaria, cómo existe una visión distorsionada de la imagen corporal, tanto en hombres como en mujeres. Los primeros presentan mayor preocupación por parecer fuertes, y las mujeres se perciben con mayor tamańo del que tienen. En ambos casos existe distorsión respecto a la realidad, y esto puede conllevar conductas alteradas. También otros estudios realizados en población físicamente activa (Damasceno, Lima, Vianna, Vianna, y Novaes, 2005) muestran cómo para los hombres el cuerpo ideal es mayor del que tienen y, por el contrario, para las mujeres el cuerpo ideal es más delgado del que tienen. Tanto en activos como en no activos, y en hombres como en mujeres, existe insatisfacción corporal (Damasceno, Lima, Vianna, Vianna, y Novaes, 2005). También existe relación entre trastornos de alimentación y distorsión de la imagen corporal (Behar y Arancibia, 2015), encontrando que un buen nivel de satisfacción con la imagen corporal disminuye la probabilidad de trastorno alimentario (Molina, Milian y Ortega, 2016).

Por otro lado, la población universitaria es un grupo especialmente susceptible, ya que la entrada en la universidad implica cambios en su estilo de vida, un alejamiento del núcleo familiar, mayor independencia, y un aumento de las relaciones con su grupo de iguales (Carrascosa, Segovia, y Monzó, 2013); además de la influencia de la publicidad y medios de comunicación que favorecen la insatisfacción corporal, y suponen un riesgo para el inicio de conductas no saludables de control de peso y trastornos de conducta alimentaria (Gonçalves y Martínez, 2014). En población universitaria existen pocos estudios en esta línea, y en concreto en población portuguesa, de ahí la importancia de este trabajo.

Una vez planteado el problema, se define como objetivo principal de este estudio: analizar y comprender las relaciones existentes entre el nivel de actividad física, la composición corporal, la percepción y la satisfacción con la imagen corporal en estudiantes universitarios del Algarbe (Portugal).

\section{Material y métodos}

\section{Participantes}

En el presente trabajo, la población objeto de estudio corresponde al alumnado inscrito en el Instituto Superior Don Afonso III (Loulé, Portugal; n=775). Participaron 400 alumnos, por lo que se asume para la validez en los resultados, un margen de error muestral del $\pm 3,4 \%$ y un nivel de confianza del $95 \%$. La muestra $(n=400)$ está constituida por $198 \mathrm{mu}$ jeres (49,5\%) y 202 hombres (50,5\%), distribuidos en cuatro grupos de edad: un primer grupo, de los 18 a los 28 años (51,8\%); el segundo grupo de los 29 a los 39 ańos (32,8\%); el tercero, de los 40 a los 50 años (11\%) y un último grupo de los 51 a los 61 ańos (4,5\%). La muestra presenta una media de edad de 30,64 ( $M=30,64 \pm 8,85$ ańos).

\section{Instrumentos}

Para el análisis de la imagen corporal se han utilizado dos instrumentos que miden tanto el componente perceptivo como el subjetivo de la imagen corporal. Para el componente perceptivo, se utilizó "Figure Rating Scale" (Stunkard et al. 1983) que consta de 9 figuras de silueta que aumenta gradualmente de tamaño; de muy delgadas (un valor de 1) a personas muy obesas (un valor de 9), las figuras 1 y 2 corresponden a personas con bajo peso, las figuras 3 y 4 a normopeso, las figuras de 5 a 7 personas obesas, y las 8 y 9 personas muy obesas. Para el componente subjetivo se utilizó "Body Shape Questionnare” (Cooper et al. 1987), validado al portugués por Vieira, Palmeira, Francisco y Teixeira (2004). Consta de 34 ítems, con una escala liker que va desde 1 (nunca) a 6 (siempre). La suma de los puntos clasifica el nivel de preocupación con la imagen corporal. Una puntuación menor que 80 se considera sin preocupación; entre 80 y 110, preocupación leve; entre 111 y 140 preocupación moderada; y mayor que 140, preocupación grave.

En cuanto al nivel de Actividad Física, se utilizó el "International Physical Activity Questionnaire”, en su versión corta, validado y traducido al Portugués por Craig et al. (2003). Recoge tres tipos de actividades: actividad leve, actividad moderada, y actividad vigorosa, con el objetivo de clasificar a los sujetos según dichos niveles: Nivel bajo (nivel inferior, los individuos que no se encuadran en los criterios de los otros dos niveles); Nivel moderado (cumple uno de los criterios: tres o más días de actividad vigorosa al menos 20 minutos, o cinco días o más de actividad de intensidad moderada, y/o caminar al menos 30 minutos al día, o actividad de intensidad moderada o vigorosa, sumando un mínimo total de 600 METS-minuto/semana); y Nivel elevado de actividad física, si cumple los criterios (al menos tres días de actividad vigorosa, reuniendo al menos $1500 \mathrm{METS}$-minutos/semana, o siete o más días de cualquier combinación de caminar, actividad moderada o vigorosa, reuniendo un mínimo total de al menos 3000 METS-minuto/semana). Para medir la composición corporal se utilizó una balanza de bioimpedancia modelo OMRON HBF - 510W, para establecer: el peso; el porcentaje de Masa Grasa (\%MG); de Masa Muscular (\%MM); y el valor de grasa visceral o perímetro de cintura (PC). Para la medida de la altura se utilizó una cinta métrica.

\section{Procedimiento}

Para la recogida de datos se solicitó previamente una autorización por escrito, tanto a la dirección del centro universitario, 
como al profesorado implicado, y a los participantes. Se preparó al personal responsable de la recogida de datos con formación específica sobre los instrumentos que se utilizaron. El alumnado fue seleccionado en las aulas de clase, en presencia de su profesor/a, donde se presentó el objeto de estudio, los criterios de inclusión y exclusión, y se concertó una cita para llevar a cabo la recogida de datos en un laboratorio de Actividad Física y Salud. La recogida de datos fue voluntaria y confidencial, respetándose el carácter anónimo de los mismos.

\section{Análisis estadístico}

Se llevó a cabo un análisis descriptivo básico de tendencia central y dispersión de las distintas variables, y de porcentaje en aquellos casos que fue necesario. Se efectuaron las pertinentes pruebas de normalidad y homocedasticidad para asegurar la homogeneidad de la varianza, obstándose por la aplicación de estadísticos paramétricos. Para el análisis de las diferencias por sexo se ha utilizado la prueba de la $\mathrm{T}$ de Student para muestras independientes. Por otro parte, se realizó un análisis de varianza (ANOVA) de un factor, para el estudio de las relaciones entre los diferentes grupos de edad. Finalmente, para analizar las relaciones entre las variables Composición Corporal (peso, altura, índice de masa corporal, porcentaje de masa grasa y perímetro de cintura) y la Imagen Corporal, se utilizó el coeficiente de correlación de Pearson. Los análisis estadísticos se realizaron con la versión 15 del programa SPSS para Windows.

\section{Resultados}

\section{Composición Corporal}

El índice de masa corporal (IMC) según los valores de la Organización Mundial de la Salud, se encuentra dentro del rango de normalidad (18,50-24,99), aunque en el caso de los hombres están cerca de sobrepasar este margen y llegar a 25 que se corresponde con la categoría de sobrepeso. El porcentaje de masa grasa (MG) para este rango de edad, se sitúa dentro del normal para las mujeres (21,0-32,9\%), y elevado para los hombres (20,0-24,9\%) como se puede observar en la Tabla 1. En cuanto al porcentaje de masa muscular (MM), siguiendo los mismos valores de referencia anteriores, ambos géneros se encuentran dentro de rangos de "normalidad", siendo éste para las mujeres 24,3-30,3\%, y para los hombres 33,3-39,3\%. Por último, el perímetro de cintura también está dentro de lo normal (entre 1 y 9), tanto para las mujeres $(5,12)$, como para los hombres $(7,18)$.
Tabla 1. Resultados del test $\mathrm{T}$, relativos a las diferencias entre las medias de los parámetros de composición corporal según el sexo

\begin{tabular}{llccccccc}
\hline & & N & Media & $\begin{array}{c}\text { Desviación } \\
\text { Típica }\end{array}$ & gl & t & $p$ \\
\hline Peso & Femenino & 198 & $\mathbf{6 2 , 4 7}$ & 10,07 & 398 & $-13,90$ & 0,000 \\
& Masculino & 202 & 76,61 & 10,25 & & & \\
IMC & Femenino & 198 & 23,60 & 3,58 & 398 & $-3,18$ & 0,002 \\
& Masculino & 202 & 24,95 & 4,79 & & & \\
\%MG & Femenino & 198 & 32,28 & 7,55 & 398 & 13,40 & 0,000 \\
& Masculino & 202 & 22,81 & 6,54 & & & \\
\%MM & Femenino & 198 & 28,95 & 4,50 & 398 & $-19,21$ & 0,000 \\
& Masculino & 202 & 37,72 & 4,61 & & & \\
PC & Femenino & 198 & 5,12 & 2,93 & 398 & $-6,55$ & 0,000 \\
& Masculino & 202 & 7,18 & 3,33 & & & \\
\hline
\end{tabular}

Conforme aumenta la edad, también lo hace la media de peso, el índice de masa corporal, el \% de masa grasa, el perímetro de cintura, y disminuye la masa muscular.

Tabla 2. Resultados del Test ANOVA, composición corporal según la edad.

\begin{tabular}{cccccccc}
\hline Edad & & $\mathrm{N}$ & Media & $\begin{array}{c}\text { Desviación } \\
\text { típica }\end{array}$ & gl & $\mathrm{F}$ & $P$ \\
\hline IMC & $18-28$ & 207 & 23,53 & 4,80 & 396 & 9,60 & 0,000 \\
& $29-39$ & 131 & 24,31 & 3,22 & & & \\
& $40-50$ & 44 & 26,95 & 3,27 & & & \\
& $51-61$ & 18 & 26,22 & 4,02 & & & \\
& Total & 400 & 24,28 & 4,28 & & & \\
\%MG & $18-28$ & 207 & 25,63 & 8,23 & 396 & 8,77 & 0,000 \\
& $29-39$ & 131 & 28,65 & 7,86 & & & \\
& $40-50$ & 44 & 30,86 & 9,60 & & & \\
& $51-61$ & 18 & 32,23 & 7,81 & & & \\
& Total & 400 & 27,49 & 8,50 & & & \\
\%MM & $18-28$ & 207 & 34,84 & 6,73 & 396 & 9,23 & 0,000 \\
& $29-39$ & 131 & 32,32 & 5,51 & & & \\
& $40-50$ & 44 & 31,18 & 5,44 & & & \\
& $51-61$ & 18 & 29,70 & 4,57 & & & \\
GV & Total & 400 & 33,38 & 6,32 & & & \\
& $18-28$ & 207 & 5,01 & 2,26 & 396 & 27,99 & 0,000 \\
& $29-39$ & 131 & 6,73 & 3,75 & & & \\
& $40-50$ & 44 & 8,43 & 3,08 & & & \\
& $51-61$ & 18 & 9,66 & 4,18 & & & \\
& Total & 400 & 6,16 & 3,30 & & & \\
\hline
\end{tabular}




\section{Imagen Corporal (Componente Perceptivo)}

En cuanto a la silueta más apropiada para la mujer, tanto los hombres como las mujeres coinciden en seleccionar la silueta número 3 , aunque con tendencia a aproximarse a la silueta 4 (sexo femenino: $M=3,82$; $D T=0,69$; sexo masculino: $M=3,65$; $\mathrm{DT}=0,70)$. Estas diferencias son estadísticamente significativas $(\mathrm{t}=2,352 ; p=0,019)$.

Los hombres $(M=4,44 ; D T=1,38)$ y las mujeres $(M=4,46$; $\mathrm{DT}=1,50)$ están de acuerdo, en cuanto a su Imagen Corporal Real (percepción de su propio tamańo), indicando la silueta número 4 para ello (las diferencias entre estos valores son estadísticamente significativas $-\mathrm{t}=0,166 ; p=0,868$ ).

Cuando se pregunta con relación a la Imagen Corporal Ideal, o sea, la silueta que les gustaría tener, aquí los hombres y las mujeres no están de acuerdo. Las mujeres seleccionan la silueta $3(\mathrm{M}=3,22 ; \mathrm{DT}=0,80)$, y los hombres la silueta 4 $(\mathrm{M}=4,07 ; \mathrm{DT}=0,88)$. En este aspecto de la Imagen Corporal, los resultados indican que estas diferencias son estadísticamente significativas $(\mathrm{t}=-10,075 ; p<0,001)$.
En cuanto a la Satisfacción/Insatisfacción con la Imagen Corporal, los resultados indican que ambos sexos muestran insatisfacción, consideran que su imagen corporal real es superior a la imagen corporal ideal (la que les gustaría tener). Aun así, las mujeres presentan valores medios superiores $(M=1,24 ; D T=1,45)$ en comparación con los hombres $(\mathrm{M}=0,37 ; \mathrm{DT}=1,38)$, y esta diferencia es estadísticamente significativa $(t=6,174 ; \mathrm{p}<0,001)$.

Finalmente, en cuanto a la preocupación con la Imagen Corporal (componente subjetivo), se verifican diferencias significativas entre hombres y mujeres $(\mathrm{t}=10,243 ; p<0,001)$, ya que las mujeres tienen valores más elevados en este componente $(\mathrm{M}=2,27 ; \mathrm{DT}=1,12)$, manifestando "Preocupación leve", aunque el valor medio de los hombres ( $M=1,34$; $\mathrm{DT}=0,64)$, indica que estos también tienen Preocupación con la Imagen Corporal (Tabla 3). El instrumento utilizado (FRS) consta de 9 figuras de silueta que van aumentando progresivamente de tamańo, de muy delgada (valor 1) a muy obesa (valor 9).

Tabla 3. Resultados del Test t, para la dimensión Imagen Corporal, de acuerdo con el sexo.

\begin{tabular}{|c|c|c|c|c|c|c|c|}
\hline & & $\mathrm{N}$ & Media & Desv Típica & $\mathrm{gl}$ & $\mathrm{t}$ & $\mathrm{p}$ \\
\hline \multirow[t]{2}{*}{ Mujer ideal } & Femenino & 198 & 3,82 & 0,69 & 398 & 2,352 & 0,019 \\
\hline & Masculino & 202 & 3,65 & 0,70 & & & \\
\hline \multirow[t]{2}{*}{ ICReal } & Femenino & 198 & 4,46 & 1,50 & 398 & 0,166 & 0,868 \\
\hline & Masculino & 202 & 4,44 & 1,38 & & & \\
\hline \multirow[t]{2}{*}{ ICIdeal } & Femenino & 198 & 3,22 & 0,80 & 398 & $-10,075$ & 0,000 \\
\hline & Masculino & 202 & 4,07 & 0,88 & & & \\
\hline \multirow{2}{*}{$\begin{array}{l}\text { Satisfacción/ } \\
\text { Insatisfacción } \\
\text { IC }\end{array}$} & Femenino & 198 & 1,24 & 1,45 & 398 & 6,174 & 0,000 \\
\hline & Masculino & 202 & 0,37 & 1,38 & & & \\
\hline \multirow[t]{2}{*}{ Preocupación IC } & Femenino & 198 & 2,27 & 1,12 & 398 & 10,243 & 0,000 \\
\hline & Masculino & 202 & 1,34 & 0,64 & & & \\
\hline
\end{tabular}

Con respecto a la discrepancia entre la IC Real (silueta seleccionada para el "propio tamaño") y la IC Ideal (silueta seleccionada para el "tamańo que le gustaría tener"), que co- rresponde a la "Satisfacción/Insatisfacción con Imagen Corporal”, en la Tabla 4, son presentados los resultados descriptivos de esta variable. 
Tabla 4. Resultados descriptivos para el componente perceptivo de la Imagen Corporal - Satisfacción/Insatisfacción con la Imagen Corporal.

\begin{tabular}{cccc}
\hline & & Número de participantes & Porcentaje \\
\hline & $(2)$ & 15 & 3,80 \\
INSATISFECHO & $(1)$ & 45 & 11,30 \\
CON IC & $(3)$ & 1 & 0,30 \\
(ICreal inferior icideal) & $(7)$ & 1 & 0,30 \\
\hline ICreal = ICideal & SATISFECHO CON IC & 107 & 26,80 \\
\hline & $(1)$ & 122 & 30,50 \\
INSATISFECHO CON IC & $(2)$ & 73 & 18,30 \\
(ICreal superior icideal) & $(3)$ & 20 & 5,00 \\
\hline
\end{tabular}

Una gran parte de los participantes de la muestra $(30,50 \%)$ manifiesta insatisfacción con su imagen corporal por exceso en una silueta, o sea, la seleccionada para el propio tamaño (ICReal) es mayor en una silueta que la ICIdeal (tamaño que le gustaría tener). Pero a pesar de esta insatisfacción, se verifica que el $(26,80 \%)$ están satisfechos con su imagen corporal, ya que la silueta correspondiente a la ICReal es la misma que presentan como ideal (diferencia igual a cero, entre la ICReal y la ICIdeal). En cuanto al resto, destacan los siguientes resultados: el 18,30\% están insatisfechos por exceso en dos siluetas (la diferencia entre la ICReal y la ICIdeal es de +2 ). Por el contrario, el 11,30\% están insatisfechos por la delgadez, o sea, la silueta que seleccionan como Ideal es superior a la que perciben como la real; un 5,00\% están insatisfechos por exceso con una diferencia de 3 siluetas entre a ICReal y la ICIdeal; un 3,80\% manifiestan insatisfacción por la delgadez, con la diferencia de dos siluetas; y un 2,30\% están insatisfechos por exceso en 5 siluetas.

\section{Imagen corporal (Componente Subjetivo-Preocupación con la Imagen Corporal)}

El cuestionario "Body Shape Questionnare" se valora en una escala de 1 (nunca) a 6 (siempre), donde una puntuación menor que 80 se considera "sin preocupación", entre 80 y 110 "preocupación leve", entre 110 y 140 "preocupación moderada”, y mayor que 140 "preocupación grave". Conforme indican los resultados presentados en la Tabla 5, los participantes de la muestra, en su mayoría (53,50\%), no presentan ninguna preocupación con la imagen corporal. En cuanto al número de participantes que manifiestan una preocupación leve, el porcentaje es del 24,00\%. Los que manifiestan preocupación moderada (11,50\%), y grave son el $11,00 \%$.
Tabla 5. Resultados descriptivos para el componente subjetivo de la Imagem Corporal - Preocupación con la Imagen Corporal.

\begin{tabular}{ccc}
\hline & Número de individuos & Porcentaje \\
\hline Sin preocupación & 214 & 53,50 \\
Preocupación Leve & 96 & 24,00 \\
Preocupación Moderada & 46 & 11,50 \\
Preocupación Grave & 44 & 11,00 \\
\hline
\end{tabular}

\section{Nivel de Actividad Física}

Para esto, se ha utilizado el "International Physical Activity Questionnaire". Como se puede comprobar en la Tabla 6, la mayoría de los participantes de la muestra (40,80\%) tienen un nivel bajo de actividad física; el 34,00\% tienen un nivel alto, y el 25,30\% un nivel moderado.

Tabla 6. Resultados de actividad física.

\begin{tabular}{ccc}
\hline & Número de individuos & Porcentaje \\
\hline Bajo & 163 & 40,80 \\
Moderado & 101 & 25,30 \\
Elevado & 136 & 34,00 \\
Total & 400 & 100,00 \\
\hline
\end{tabular}

Los hombres presentan valores superiores (Nivel Moderado: $\mathrm{M}=2,07 ; \mathrm{DT}=0,86$ ), en comparación con las mujeres (Nivel Bajo/Moderado: $\mathrm{M}=1,79$; $\mathrm{DT}=0,84$ ), siendo esta diferencia estadísticamente significativa $(\mathrm{t}=-3,240 ; p=0,001)$ (Tabla 7$)$. 
Tabla 7. Test t, relativos al análisis de Actividad Física, en función del sexo.

\begin{tabular}{llcccccc}
\hline & & $\mathrm{N}$ & Media & Desviación-Típica & $\mathrm{gl}$ & $\mathrm{t}$ & $\mathrm{p}$ \\
\hline \multirow{2}{*}{ Nivel de Actividad Física } & Femenino & 198 & 1,79 & 0,84 & 398 & $-3,240$ & 0,001 \\
& Masculino & 202 & 2,07 & 0,86 & & & \\
\hline
\end{tabular}

Tabla 8. ANOVA de Atividad Física en función de la edad.

\begin{tabular}{ccccccc}
\hline & $\mathrm{N}$ & Media & Desviación típica & $\mathrm{gl}$ & $\mathrm{F}$ & $p$ \\
\hline $18-28$ & 207 & 2,18 & 0,84 & 396 & 13,333 & 0,000 \\
$29-39$ & 131 & 1,72 & 0,82 & & & \\
$40-50$ & 44 & 1,52 & 0,76 & & & \\
$51-61$ & 18 & 1,67 & 0,68 & & & \\
Total & 400 & 1,93 & 0,86 & & & \\
\hline \hline
\end{tabular}

En cuanto a la edad, en la Tabla 8 se comprueba que sólo los participantes más jóvenes (intervalo entre 18 y 28 ańos) presentan un nivel moderado de actividad física $(M=2,18$, DT=0,84), teniendo los restantes (intervalo entre 29 a 61 años) un nivel bajo/moderado de actividad física. Este análisis reveló diferencias estadísticamente significativas $(\mathrm{t}=13,333$; $p<0,001)$.

\section{Correlación entre Composición Corporal e Imagen Corporal}

Se realiza a continuación el análisis del peso, el IMC y \% MG en relación a la Imagen Corporal.

Tabla 9. Correlación de Pearson entre las variables Composición Corporal (Peso, IMC, \%MG) y la Imagen Corporal.

\begin{tabular}{|c|c|c|c|c|}
\hline Peso & ICReal & ICIdeal & Satisfacción/Insatisfacción & Preocupación \\
\hline Coeficiente Correlación & $0,387^{* *}$ & $0,322^{* *}$ & $0,175^{* *}$ & $-0,078$ \\
\hline Sig. (2-tailed) & 0,000 & 0,000 & 0,000 & 0,119 \\
\hline \multicolumn{5}{|l|}{ IMC } \\
\hline Coeficiente Correlación & $0,411^{* *}$ & 0,090 & $0,345^{* *}$ & $0,149^{* *}$ \\
\hline Sig. (2-tailed) & 0,000 & 0,073 & 0,000 & 0,003 \\
\hline \multicolumn{5}{|l|}{$\% \mathrm{MG}$} \\
\hline Coeficiente Correlación & $0,258^{* *}$ & $-0,236^{* *}$ & $0,418^{* *}$ & $0,452^{* *}$ \\
\hline Sig. (2-tailed) & 0,000 & 0,000 & 0,000 & 0,000 \\
\hline
\end{tabular}

$\mathrm{n}=400 ;{ }^{* *}$ Correlación significativa (nivel 0,01$)$

De acuerdo con los resultados presentados en la Tabla 9, se observan correlaciones significativas (nivel 0,01), en los siguientes parámetros de la Imagen Corporal: ICReal $(\mathrm{p}<0,001)$, ICIdeal $(\mathrm{p}<0,001)$ y Satisfacción/Insatisfacción con la Imagen Corporal $(\mathrm{p}<0,001)$, a través del análisis del coeficiente de correlación de Pearson. Así, se encontraron evidencias estadísticas de correlación positiva entre las variables Composición Corporal (Peso) e Imagen Corporal Real $(r=0,387)$, Imagen Corporal Ideal $(r=0,322)$ y Satisfacción/ Insatisfacción con la Imagen Corporal $(r=0,175)$. Significan estos datos que, cuanto mayor es el peso, mayor la silueta que los participantes presentan como real e ideal. Con relación a la satisfacción/insatisfacción, cuanto mayor es el peso, más insatisfacción con su Imagen Corporal. Con relación a la Preocupación con la Imagen Corporal, existe una correlación negativa, con un coeficiente de $r=-0,078$, lo que significa que cuanto más peso presentan los participantes, menos preocupación muestran con su Imagen Corporal.

Con relación al IMC y el coeficiente de correlación de Pearson, se observó la existencia de correlación significativa (nivel 0,01), entre las categorías de IMC, la Imagen Corporal Real ( $\mathrm{p}<0,001)$, la Satisfacción/Insatisfacción ( $\mathrm{p}<0,001)$ y la Preocupación con la Imagen Corporal $(p=0,003)$. Existen evidencias estadísticas de correlación positiva con todos los parámetros de la Imagen Corporal considerados, lo que significa que cuanto mayor es el IMC, mayor la silueta que los participantes indican como Real $(r=0,411)$ e Ideal $(r=0,090)$, y más insatisfechos $(r=0,345)$ y preocupados $(r=0,149)$ están con su Imagen Corporal. En cuanto al porcentaje de Masa Grasa, los resultados parecen indicar que existe una correlación positiva con la Imagen Corporal Real $(r=0,258 ; p<0,001)$, con la Satisfacción/Insatisfacción $(r=0,418$; $p<0,001)$ y con la Preocupación $(r=0,452 ; p<0,001)$, siendo negativa la que se señala como Imagen Corporal Ideal $(r=-0,236$, $\mathrm{p}<0,001$ ). Todas estas correlaciones son significativas (nivel 0,01). Así, en cuanto al porcentaje de masa grasa, se puede decir que los resultados apuntan que cuanto mayor este valor, mayor también será la silueta que representa la Imagen Corporal Real, mayor será la insatisfacción con la Imagen Corporal y la preocupación que los participantes tienen con su Imagen Corporal. Además, cuanto mayor será el \%MG, menor será la IC ideal. 


\section{Correlación entre Composición Corporal y Nivel de Actividad Física}

Manteniendo la variable Composición Corporal, continuamos este análisis del parámetro "\% Masa Muscular", con idea de entender la relación entre éste y el nivel de actividad física.

Tabla 10. Correlación de Pearson entre las variables Composición Corporal y el Nivel de Actividad Física.

\begin{tabular}{lcccl}
\hline \hline NAF & Peso & IMC & $\% M G$ & $\% M M$ \\
\hline Coeficiente Correlación & $-0,034$ & $-0,209^{* *}$ & $-0,252^{* *}$ & $0,234^{* *}$ \\
Sig. (2-tailed) & 0,497 & 0,000 & 0,000 & 0,000 \\
\hline n=400; ${ }^{* *}$ Correlación significativa (nivel 0,01$)$ & &
\end{tabular}

Conforme indican los resultados de la Tabla 10, se comprueba que las correlaciones entre el nivel de actividad física y el peso $(r=-0,034)$, IMC $(r=-0,209)$ y $\%$ MG $(r=-0,252)$ son negativas, lo que significa que cuanto mayor sean los valores de actividad física, menor será el peso, el IMC y el \%MG. En cuanto al \%MM $(r=0,234)$ sucede lo contrario, cuanto más activa es la persona, más masa muscular presenta. Conforme sugieren los valores del coeficiente de Pearson, son significativas las correlaciones con el IMC, \%MG y \%MM.

\section{Correlación entre Imagen Corporal y Nivel de Actividad Física (NAF)}

Tabla 11. Correlación de Pearson entre las variables Nivel de Actividad Física e Imagen Corporal.

\begin{tabular}{lllll}
\hline \hline NAF & ICReal & ICIdeal & $\begin{array}{l}\text { Satisfacción/ } \\
\text { Insatisfacción }\end{array}$ & Preocupación \\
\hline $\begin{array}{l}\text { Coeficiente } \\
\text { Correlación }\end{array}$ & $-0,386^{* *}$ & $-0,080$ & $-0,354^{* *}$ & $-0,408^{* *}$ \\
Sig. (2-tailed) & 0,000 & 0,109 & 0,000 & 0,000 \\
\hline
\end{tabular}

$\mathrm{n}=400 ;{ }^{* *}$ Correlación significativa (nivel 0,01 )

Del análisis de los coeficientes de Pearson, en la Tabla 11 se observa la existencia de correlaciones significativas (nivel 0,01 ), entre la variable nivel de actividad física y la Imagen Corporal Real $(r=-0,386, p<0,001)$, Satisfacción/Insatisfacción $(r=-0,354 ; p<0,001)$ y la Preocupación con la Imagen Corporal $(r=-0,408 ; p<0,001)$. Se encuentran evidencias estadísticas de correlación negativa entre el nivel de actividad física de los participantes y todos los parámetros de la Imagen Corporal evaluados. Esto significa que cuanto más activos son los participantes de la muestra, menor será la silueta seleccionada para la ICReal, Ideal, más satisfechos y menos preocupados con su Imagen Corporal.

\section{Discusión}

Como ya se ha comentado, el objetivo principal de este estudio era analizar y comprender las relaciones existentes entre el nivel de actividad física, la composición corporal, la percepción y la satisfacción con la imagen corporal en estudiantes universitarios portugueses. En relación a la percepción de la imagen corporal que tienen hombres y mujeres, los resultados de la presente investigación coinciden con Labre (2002), donde hombres y mujeres están de acuerdo con la figura más adecuada para el hombre (4) y la mujer normal (3), para una IC real (4) y para la satisfacción/insatisfacción (insatisfechos por exceso), confirmando lo que plantean sobre el ideal de belleza en el que la tendencia es un patrón cultural de hombre musculado, con elevado grado de hipertrofia y niveles bajísimos de grasa. También Murnen (2011), mantiene la idea de que, en la cultura occidental, el concepto de belleza está asociado a la juventud, un cuerpo delgado es atractivo para las mujeres, y un cuerpo voluminoso y musculado para los hombres. Por tanto, las mujeres y los hombres difieren en la IC Real, manifestando que las mujeres desean una silueta por debajo de la que desean los hombres. O sea, ellas se perciben una silueta por encima de la que prefieren, lo que no ocurre con los hombres. También González, De la Montaña, Míguez, Gonzalez, y Velo (2003) resaltan la preocupación por la apariencia corporal, de la que no están exentos hombres y mujeres, hagan o no actividad física. Aun así, parece haber una relación positiva entre ejercicio físico y tener una evaluación más ajustada a la realidad sobre el propio cuerpo (Marques, 2003; Oliveira, 2009, Womack, 2010), y así mayor satisfacción con el mismo (Grogan et al., 2014).

Destaca también la diferencia en cuanto a los valores de preocupación con la IC, donde las mujeres están levemente preocupadas y los hombres no. Estos resultados coinciden con los de Cash (2011) que mantiene que el cuerpo delgado como representación ideal está reforzado a través de los medios de comunicación, y potencia el aumento de la insatisfacción con la imagen corporal, teniendo repercusiones negativas para la salud (por ejemplo, trastornos de alimentación). En esta línea, Raich (2004) añade que estos medios incentivan las comparaciones sociales.

Respecto a la edad, parece existir una tendencia de insatisfacción o preocupación con la IC por parte de los sujetos más jóvenes. Este resultado coincide con la idea de Raich (2001), que mantiene que la imagen corporal se va construyendo en diferentes etapas de la vida.

El perfil de los alumnos que practican más actividad física son los hombres, los más jóvenes y solteros, siendo el nivel de la muestra general considerado como bajo y moderado. Estos datos coinciden con diversos estudios, que han señalado la entrada en la universidad como un momento en el que aumenta la tasa de abandono de práctica de actividad física 
(Gómez-López, Granero y Extremera, 2010; Castillo y Giménez, 2011). Existen estudios similares donde el sedentarismo es más común entre las mujeres, los adultos mayores y los sujetos con menos nivel sociocultural (Crespo et al., 2000; Gómez-López, Granero y Extremera, 2010; Pitanga y Lessa, 2005; Sallis y Owen, 1998; y Sallis y Saelens, 2000).

En cuanto a la composición corporal, el 58,8\% de los participantes se encuentran en la categoría de normopeso y el 33 $\%$ con sobrepeso, muy similar a los datos encontrados en el estudio de Salazar, Feu, Vizuete y de la Cruz (2013) en Méjico, en el que el porcentaje de sujetos normopeso es de 52,7 \% y el de sobrepeso es del 33,3 \%. Por el contrario, el porcentaje de obesos encontrado es más bajo en este trabajo $(6,3 \%)$ frente a los estudiantes del estudio mejicano $(9,1 \%)$.

El análisis de esta relación entre composición corporal y nivel de actividad física indica que los sujetos que son más activos, son los que se incluyen en una categoría de normal y delgadez de IMC, siendo los obesos los menos activos. Los individuos más activos de la muestra seleccionan las siluetas 2 y 3 como las adecuadas para el hombre normal, y los menos activos señalan siluetas mayores. Lo mismo sucede con la IC Real, esto es, los más activos consideran que su cuerpo corresponde a figuras más delgadas de lo que seńalan los menos activos. Coincide con esto los resultados de Marques (2003), donde se relaciona la práctica regular de actividad física y la valoración que las personas hacen de su cuerpo, verificando que la práctica adecuada de ejercicio físico, considerada como una opción de inversión en salud corporal, contribuye significativamente en valores más precisos y positivos de los participantes con relación a su propio cuerpo, lo que proporciona mayores niveles de satisfacción corporal. También Oliveira (2009) mantiene que la práctica de actividad física, no sólo aumenta el interés y el nivel de satisfacción con el cuerpo, sino también parece contribuir a una valoración más rigurosa y positiva de la imagen corporal; así como a una mayor y mejor dedicación al cuerpo. En esta línea, Coqueiro, Petroski, Pelegrini y Barbosa (2008), en un trabajo que analizaba la insatisfacción corporal en universitarios, mostraron resultados que indicaban una asociación significativa ente el IMC y la insatisfacción con la imagen corporal. Los resultados mostraron que un gran número de universitarios están insatisfechos con la propia imagen corporal, y que las mujeres, de manera general, desean reducir el tamaño de su silueta corporal. En cuanto a los hombres, el deseo es tener un cuerpo más grande y voluminoso. Esta diferencia puede ser explicada por el hecho de que las mujeres sobreestiman la imagen corporal y los hombres la subestiman.

Considerando la correlación entre los parámetros de IC y $\mathrm{NAF}$, todas tienen naturaleza negativa, siendo por eso inversamente proporcionales. Ello significa que cuanto más activo es el sujeto, menores son las siluetas seleccionadas para la IC Real, más satisfechos y menos preocupados con su IC, y muestran que la actividad física puede tener un papel positivo en la imagen corporal de los sujetos.

\section{Conclusiones}

Respondiendo al objetivo inicial se puede afirmar que existe una relación inversa entre la mayoría de los parámetros de composición corporal e imagen corporal. Esto es, los sujetos que se identifican con las siluetas más grandes son los que están más insatisfechos por el deseo de tener menos peso, y más preocupados con su imagen corporal, y además son menos activos físicamente. Los estudiantes más activos son los que presentan valores más saludables de composición corporal, y de imagen corporal, y además son los más satisfechos y menos preocupados por la misma.

Se concluye este trabajo indicando alguna línea o perspectiva futura de investigación. Los estudios descriptivos sobre actividad física en universitarios son suficientes, por lo que deberíamos seguir avanzando y pasar a realizar y evaluar programas específicos de intervención que fomenten la práctica de actividad física en los jóvenes estudiantes. Otra línea de investigación de interés en este colectivo debería orientarse (diferenciando por género) al estudio sobre la imagen y los estereotipos que el deporte trasmite en los medios de comunicación.

\section{Referencias bibliográficas}

1. Behar, R., \& Arancibia, M. (2015). Trastornos de la imagen corporal: anorexia nerviosa versus anorexia inversa (trastorno dismórfico muscular). Revista mexicana de trastornos alimentarios, 6(2), 121-128.

2. Borrego Balsalobre, F. J., López Sánchez, G. F. \& Díaz Suárez, A. (2012). Physical condition influence in self-concept of a teens group of Alcantarilla town. Cuadernos de Psicología del Deporte, 12(S2), 57-62.

3. Borrego Balsalobre, F. J., López Sánchez, G. F. \& Díaz Suárez, A. (2014). Relationships between physical fitness and physical self-concept in Spanish adolescents. Procedia: Social and Behavioral Sciences, 132, 343-350. http://dx.doi.org/10.1016/j.sbspro.2014.04.320

4. Burgess, G., Grogan, S., \& Burwitz, L. (2006). Effects of a 6-week aerobic dance intervention on body image and physical self-perceptions in adolescent girls. Body image, 3(1), 57-66.
5. Carrascosa, R. G., Segovia, P. G., \& Monzó, J. M. (2013). Valoración de la imagen corporal y de los comportamientos alimentarios en universitarios. Revista de Psicopatología y Psicología Clínica 18(1), 45-59. Universidad Nacional de Educación a Distancia (UNED). Asociación Española de Psicología Clínica y Psicopatología.

6. Cash, T. (2011). Crucial considerations in the assessment of body image. In T. Cash; A. Lind (Ed). Body image. A handbook of science, practice and prevention. Second Ediction. London. Guilford Press.

7. Cash, T., \& Pruzinsky, T. (1990). Body Images: development, deviance and change. New York: The Guilford Press.

8. Castillo, E.; \& Giménez, F.J. (2011). Hábitos de práctica de actividad física del alumnado de la Universidad de Huelva. Revista Internacional de Medicina y Ciencias de la Actividad Física y el Deporte, 11(41), 127-144. 
9. Castro-Sánchez, M., Zurita-Ortega, F., Chacón-Cuberos, R., Martínez-Martínez, A., Espejo-Garcés, T., \& Álvaro-González, J. I. (2015). Harmful substances and motivational climate in relation to physical activity. Health and Addictions/Salud y Drogas, 15(2), 115-126.

10. Chacón Cuberos, R., Castro Sánchez, M., Caracuel Cáliz, R., Padial Ruz, R., Collado Fernández, C., \& Zurita Ortega, F. (2016). Profiles of alcohol and tobacco use among adolescents from Andalusia in the first cycle of secondary education. Health and Addictions/Salud y Drogas, 16(2), 93-104.

11. Cooper, P., Taylor, M., Cooper, Z. \& Fairburn, C. (1987). The development and validation of the Body Shape Questionnaire. Internacional Journal Eat Disorder, 6(4), 485-94.

12. Coqueiro, R., Petroski, E., Pelegrini, A., Barbosa, A. (2008). Insatisfação com a imagem corporal: avaliação comparativa da associação com estado nutricional em universitários. Revista de Psiquiatria do Rio Grande do Sul, 30(1).

13. Craig, C. L., Marshall, A. L., Sjöström, M., Bauman, A. E., Booth, M. L., Ainsworth, B. E., ... \& Oja, P. (2003). International physical activity questionnaire: 12 -country reliability and validity. Medicine \& Science in Sports \& Exercise, 35(8), 81-95.

14. Crespo, C. J., Smit, E., Andersen, R. E., Carter-Pokras, O., \& Ainsworth, B. E. (2000). Race/ethnicity, social class and their relation to physical inactivity during leisure time: results from the Third National Health and Nutrition Examination Survey, 1988-1994. American journal of preventive medicine, 18(1), 46-53.

15. Damasceno, V., Lima, J., Vianna, J., Vianna, V., \& Novaes, J. (2005). Tipo físico ideal e satisfação com a imagem corporal de praticantes de caminhada. Revista Brasileira de Medicina do Esporte, 11(3), 181-186.

16. Espejo Garcés, T., Martínez Martínez, A., Chacón Cuberos, R., Zurita Ortega, F., Castro Sánchez, M., \& Cachón Zagalaz, J. (2017). Alcohol consumption and physical activity in adolescents from rural environment. Health and Addictions/Salud y Drogas, 17(1), 97-105.

17. Gómez-López, M.; Granero, A. \& Extremera, A. (2010). Perceived barriers by universitiy students in the practice of physical activities. Journal of sports science and medicine, 9, 374-381.

18. Gonçalves, V. O., \& Martínez, J. P. (2014). Imagen corporal y percepción de la influencia de los medios de comunicación: diferencias de género en una muestra de adolescentes. Revista Interação, 39(3), 461-478.

19. González Carnero, J., De la Montańa Miguélez, J., Míguez Bernárdez, M., Gonzalez Tesouro, M. P., \& Velo Cid, C. (2003). Comparación de la calidad nutricional de los menús ofertados, en la temporada de invierno y verano, en un comedor universitario. Alimentaria, (348), 51-56.

20. Grogan, S., Williams, A., Kilgariff, S., Bunce, J., Heyland, J. S., Padilla, T., ... \& Davies, W. (2014). Dance and body image: young people's experiences of a dance movement psychotherapy session. Qualitative Research in Sport, Exercise and Health, 6(2), 261-277.

21. Hart, E. (2000). Assessing body image. En K.A.Tritschler, Practical measurement and assessment. pp. 409-437. Baltimore: Lippincott Williams \& Wilkins.

22. Hausenblas H., \& Fallon E. (2006). Exercise and body image: a metaanalysis. Psychol Health; 21, 33-47.

23. IPAQ (2005). Guidelines for data processing and analysis of the International Physical Activity Questionnaire (IPAQ). Short and Long Forms. Recuperado de http://www.ipaq.ki.se/scoring.pdf.

24. Krane, V., Waldron, J., Stiles-Shipley, J. A., \& Michalenok, J. (2001). Relationships among body satisfaction, social physique anxiety, and eating behaviors in female athletes and exercisers. Journal of Sport Behavior, 24(3), 247.

25. Labre, M. (2002). Adolescents boys and the muscular male body ideal. Journal of Adolescents Health, 30(4), 233-242.

26. López Sánchez, G. F., Borrego Balsalobre, F. J. \& Díaz Suárez, A. (2013). Effects of a physical activity program on body composition of school children of 3-5 years. Sport TK: Revista EuroAmericana de Ciencias del Deporte, 2(2), 41-44.
27. López Sánchez, G. F., López Sánchez, L. \& Díaz Suárez, A. (2015). Body composition and heart rate variability: relations to age, sex, obesity and physical activity. Sport TK: Revista EuroAmericana de Ciencias del Deporte, 4(2), 33-40.

28. López Sánchez, G. F., Nicolás López, J. \& Díaz Suárez, A. (2016). Effects of a program of intense physical activity on the body composition of adolescents from Murcia. Sport TK: Revista EuroAmericana de Ciencias del Deporte, 5(2), 83-88.

29. López Sánchez, L., López Sánchez, G. F. \& Díaz Suárez, A. (2015). Effects of a physical activity program on the body image of schoolchildren with ADHD. Cuadernos de Psicología del Deporte, 15(2), 135-142.

30. López-Sánchez, G. F., Díaz-Suárez, A., Radzimiński, Ł., \& Jastrzębski, Z. (2017). Effects of a 12-week-long program of vigorous-intensity physical activity on the body composition of 10-and 11-year-old children. Journal of Human Sport and Exercise, 12(1), 235-244. doi:10.14198/ jhse.2017.121.19

31. Marques, A. (2003). Perceçóes de saúde, competência e imagem corporal dos jovens que frequentam estabelecimentos militares de ensino em Portugal. Dissertaçáo de Mestrado. Universidade Técnica de Lisboa. Trabajo inédito. Lisboa: Faculdade de Motricidade Humana.

32. Murnen, S. (2011). Gender and Body Images. In T. Cash, A. Linda (ed), Body image - a handbook of Science, practice and prevention. Second edition. NY and London: The Guilford Press.

33. Molina, M. D. P. U., Milian, M. P., \& Ortega, M. J. R. (2016). Conductas alimentarias de riesgo y su relación con la imagen corporal en estudiantes de enfermería. Ciencia y Cuidado, 12(2), 57-71.

34. Oliveira, T. (2009). Atividade física, desporto e imagem corporal - estudo em adolescentes com peso normal e com excesso de peso. Dissertação apresentada com vista à obtenção do $2^{\circ}$ ciclo em Atividade Física e Saúde. Trabajo inédito. Universidade do Porto: Faculdade de Desporto.

35. Pitanga, F., Lessa, I. (2005) Prevalência e fatores associados ao sedentarismo no lazer em adultos. Saúde Pública, 21(3), 870-877.

36. Raich, R. (2001). Imagen Corporal - conocer y valorar el propio cuerpo. Lisboa. Pirámide.

37. Raich, R. (2004). Una perspectiva desde la psicología de la salud de la Imagen Corporal. Avances en Psicología Latinoamericana, 22, 15-27.

38. Russo, R. (2005). Imagem corporal: Construção através da cultura do belo. Movimento \& Perceção, 5(6), 80-90.

39. Salazar, Z. (2008). Adolescencia e imagen corporal en la época de la delgadez. Reflexiones, 87(2), 67-80. Universidad Costa Rica. Costa Rica.

40. Salazar C., C.M.; Feu, S., Vizuete, M. \& De la Cruz-Sánchez, E. (2013). IMC y actividad física de los estudiantes de la Universidad de Colima. Revista Internacional de Medicina y CC de la Actividad Física y del Deporte, 13(51), 569-584.

41. Sallis, J.; \& Owen, N. (1998): Determinants of Physical Activity. Behavioral Medicine \& Health Psychology, 7, 110-132.

42. Sallis, J., \& Saelens, B. (2000): Assessment of physical activity by selfreport: status, limitations, and future Directions. Research Quartely for Exercise and Sport, 71(2), 1-14.

43. Stunkard A., Sorenson T., \& Schulsinger F. (1983) Use of the Danish adoption register for the study of obesity in thinness. In: Kety $S$, Rowland LP, Sidman RL, \& Matthysse SE (Eds.) The genetics of neurological and psychiatric disorders. New York: Raven; p. 115-20.

44. Teixeira P., Going, S., \& Houtkooper, L., Cussler, E., Metcalfe, L., Blew, R., et al. (2006). Exercise motivation, eating, and body image variables as predictors of weight control. Medicine Science Sports \& Exercise; 38, 179-88.

45. Vieira, P., Palmeira, A., Francisco, C., \& Teixeira, P. (2004). Validação da versão portuguesa do Questionário da Forma Corporal. Actas do $5^{\circ}$ Congresso Nacional de Psicologia da Saúde, 45-50. Lisboa.

46. Womack, A. (2010). Body Image and Exercise among older adults. Thesis submitted for the degree of master in Psychology. Sacramento. California State University. 